\title{
PREVALÊNCIA DE DEMODEX FOLLICULORUM E DEMODEX BREVIS EM UMA AMOSTRA DA POPULAÇÃO DE BOTUCATU, SÃO PAULO, BRASIL
}

\author{
N.G. Madeira e M.I.L. Sogayar
}

\begin{abstract}
Pela primeira vez no Brasil foi realizado um levantamento para se conhecer a distribuição do D. folliculorum e D. brevis no homem. Uma amostra de 100 pessoas atendida em clinica estética foi examinada, procurando-se estudar a associação entre a presença de ácaros e fatores como idade, raça $e$ sexo do hospedeiro. O material colhido da região facial dos individuos foi montado em lâminas com o meio de Berlese. Das 100 pessoas examinadas, $72 \%$ foram positivas. Dos casos positivos, $51 \%$ estavam infestados pelo D. folliculorum, $2 \%$ pelo D. brevis e 19\% apresentaram-se parasitados por ambas as espécies.
\end{abstract}

Palavras-chaves: Demodex folliculorum. Demodex brevis. Prevalência.

A família Demodicidae compreende atualmente duas espécies distintas, parasitas do homem, oD. folliculorum e $\mathrm{D}$. brevis. Ambas, são encontradas no complexo pílo-sebáceo ocupando, entretanto, nichos diferentes. $O D$. folliculorum habita os folículos pilosos e $\mathrm{O} D$. brevis é visto principalmente nas glândulas sebáceas ${ }^{4}$. A importância desses ácaros, em relação à espécie humana, vem atualmente recebendo maior atenção, com um número crescente de estudos devido à sua grande prevalência, cosmopolitismo e suspeita de causar e transmitir doenças no homem. Embora a ação patogênica destes ácaros ao homem, seja ainda causa de controvérsias, existe relato de pacientes com dermatite semelhante à rosácea apresentando grande número de parasitas na pele ${ }^{1}$. D. folliculorum foi encontrado em $80 \%$ dos pacientes com rosácea e em apenas $10 \%$ dos controles ${ }^{16}$. O estudo de 7 casos humanos com rosácea pápulo-pustular usando a imunofluorescência indireta, sugere que o $D$. folliculorum é capaz de induzir uma resposta imune que pode explich a passagem de un quadro predominantemente funcional para uma fase inflamatória da doenç̧a ${ }^{8}$. A síndrome da imunodeficiência adquirida (SIDA) propicia a proliferação de $D$. follthculorum a ponto de causar doenças $^{5}$. O uso de acaricidas é capaz de curar dermatites crônicas semelhantes à rosácea ${ }^{1315}$,

\footnotetext{
Departamento de Parasitologia/Instituto de Biociências/ Universidade Estadual Paulista/Botucatu, SP.

Endereço para correspondência: Dr. Goulart Madeira. Depto. de Parasitologia/IB/UNESP, Campus de Botucatu, Rubiāo Junior 18618-000, Botucatu, SP.

Recebido para publicação em 30/06/93.
}

como também dermatite perioral e blefarite ${ }^{6} 12$. Alguns autores têm sugerido a possibilidade desses ácaros abrigarem no seu interior agentes patogênicos e sua capacidade de transportá-los para outras regiões da pele ou para outras pessoa ${ }^{17}$.

No Brasil é grande a escassez de dados sobre a prevalência desses ácaros no homem ${ }^{13}$. O presente trabalho vem apresentar dados sobre a prevalência do $D$. folliculorum e $D$. brevis, sendo a presença deste último relatada pela primeira vez em nosso país e os resultados obtidos relacionados com à idade, sexo, raça, tipo de pele e lesões encontradas no hospedeiro.

\section{MATERIAL E MÉTODOS}

Material de 100 pessoas, 90 mulheres e 10 homens, frequentadores de clínica para limpeza de pele, foi colhido num período de 3 meses, no verão. Cada indivíduo preencheu uma ficha onde constava nome, sexo, idade, raça, tipo de pele (normal, seca e oleosa) e os tipos de lesōes encontradas (comedon, acne, caimento de cílios, inflamações de cílios e outros tipos de inflamação). Quanto à raça, todos os indivíduos tinham pele clara e 3 eram descendentes de japoneses. .O tipo de pele foi determinado por aparelho estético para essa finalidade. $O$ material foi retirado por compressão, com extrator de comedons esterilizado, após tratamento dermo-estético com auxílio de calor para abertura dos poros. Coletou-se uma amostra por indivíduo, que foi colocada em um recipiente com etanol a $70 \%$. Todas as amostras foram retiradas pelo mesmo esteticista com luvas 
Madeira NG, Sogayar MIL. Prevalência de Demodex folliculorum e Demodex brevis em uma amostra da população de Botucatu, São Paulo, Brasil. Revista da Sociedade Brasileira de Medicina Tropical 26:221-224, out-dez, 1993.

esterilizadas e foi realizada em toda a extensão da face de cada pessoa. Com uma porção de cada amostra foram preparadas 2 lâminas permanentes com Berlese, que foram examinadas em microscópio de contraste de fase. A identificação dos ácaros foi realizada por meio dos caracteres descritos por Desch e $\mathrm{col}^{4}$. Nos dados observados, o tamanho amostral do grupo masculino não permitiu aplicação consistente das técnicas estatísticas.

\section{RESULTADOS}

$\mathrm{Na}$ amostra estudada a taxa total de parasitismo foi de $72 \%$. O D. folliculorum foi a espécie prevalente, sendo encontrada em $51 \%$ dos casos. Pode-se observar que a infestação apenas por $D$. brevis ocorreu em somente $2 \%$ da amostra, enquanto que a ocorrência concomitante das espécies foi encontrada em $19 \%$ dos casos. A porcentagem de infestação nas pessoas do sexo feminino atingiu $74,4 \%$ e do sexo masculino $50 \%$ (Tabela 1 ).

Em relação à idade, verificamos que o $D$. folliculorum foi a espécie prevalente em quase todas as faixas etárias, exceto aquela de 40 a 49 anos, quando o número de infestações mistas igualou à de D. folliculorum. Parasitismo apenas por $D$. brevis foi verificado nas faixas de 14 a 19 e 40 a 49 anos. O parasitismo pelas duas espécies foi observado em todas as idades exceto entre individuos com mais de 70 anos. Com relação à taxa total de positividade verificou-se que há uma diminuição discreta do parasitismo após os 40 anos (Figura 1).

Tabela 1 - Distribuiçðo da frequência de D. folliculorı n e D. brevis na populaçđo examinada e em funçđo do sexo dos individuos parasitados.

\begin{tabular}{|c|c|c|c|c|c|c|c|c|c|c|}
\hline & \multirow{2}{*}{\multicolumn{2}{|c|}{ D. folliculorum }} & \multirow{2}{*}{\multicolumn{2}{|c|}{ D. brevis }} & \multirow{2}{*}{\multicolumn{2}{|c|}{$\begin{array}{l}\text { Ambas } \\
\text { espécies }\end{array}$}} & \multicolumn{4}{|c|}{ Total } \\
\hline & & & & & & & \multicolumn{2}{|c|}{ Positivos } & \multicolumn{2}{|c|}{ Negativos } \\
\hline & $n^{0}$ & $\%$ & $n^{0}$ & $\%$ & $n^{0}$ & $\%$ & $n^{0}$ & $\%$ & $n^{0}$ & $\%$ \\
\hline Feminino $(n=90)$ & 46 & 68,0 & 2 & 2,9 & 19 & 28,3 & 67 & 74,4 & 23 & 25,6 \\
\hline Masculino $(n=10)$ & 5 & 100,0 & 0 & 0,0 & 0 & 0,0 & 5 & 50,0 & 5 & 50,0 \\
\hline Total & 51 & 51,0 & 2 & 2,0 & 19 & 19,0 & 72 & 72,0 & 28 & 28,0 \\
\hline
\end{tabular}

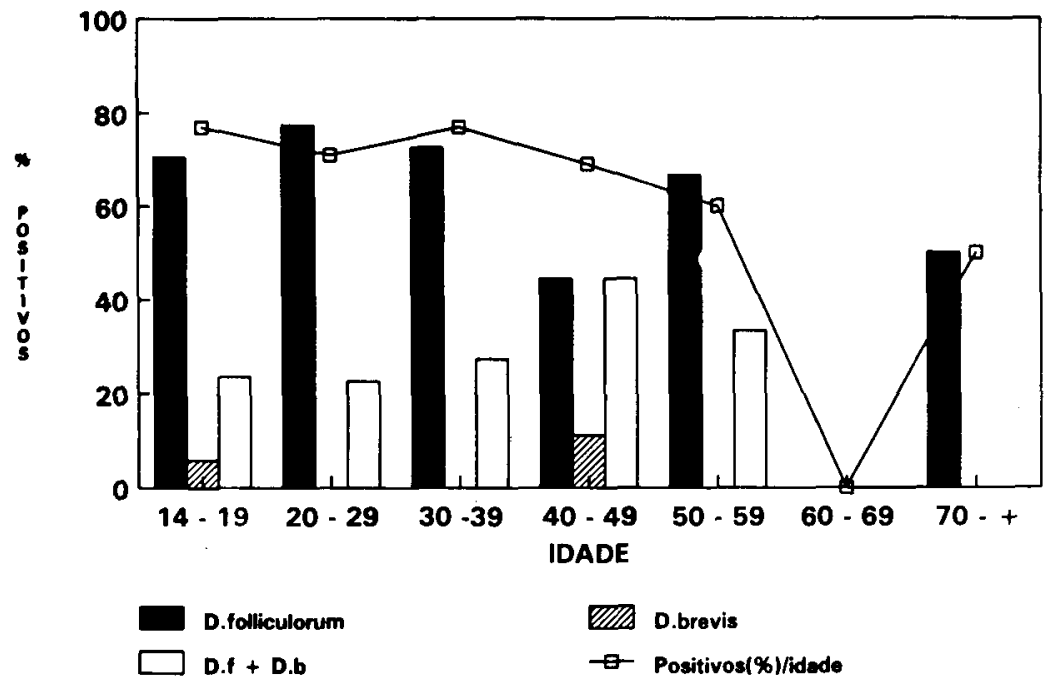

Figura 1 - Porcentagem e número totalde individuos parasitados por Demodex folliculorum $e$ D. brevis, de acordo com a faixa etária. 
Madeira NG, Sogayar MIL. Prevalência de Demodex folliculorum e Demodex brevis em uma amostra da população de Botucatu, São Paulo, Brasil. Revista da Sociedade Brasileira de Medicina Tropical 26:221-224, out-dez, 1993.

Quanto à raça, o parasitismo foi detectado apenas entre as pessoas de pele clara, que perfizeram $97 \%$ da amostra.

Levando-se em consideração o tipo de pele, $97 \%$ dos pacientes tinha pele oleosa e $3 \%$ do tipo normal, tendo sido encontrados indivíduos parasitados nos dois tipos de pele, a saber: dos 72 indivíduos parasitados 70 tinham pele oleosa e 2 tinham pele normal.

Em relação à distribuição dos ácaros e o tipo de lesões na pele, todos os indivíduos da amostra apresentaram comedons, inclusive 28 indivíduos negativos. Quanto à presença de acne somente 18 apresentaram este tipo de lesão, sendo que 7 $(36,37 \%)$ indivíduos tinham $D$. folliculorum, 4 $(26,26 \%)$ tinham infecção mista e $7(36,37 \%)$ apresentaram-se sem parasita.

\section{DISCUSSÃO}

O número de pessoas parasitadas observadas no presente estudo (72\%) foi mais elevado do que o observado em Nova York $(54,9 \%)^{14}$. Esta diferença possivelmente possa estar relacionada à amostra utilizada, uma vez que em Nova York as pessoas não estavam sob cuidados dermatológicos, o que leva à suspeitar de que a existência de ácaros na pele, possa ter alguma relação com a procura desses cuidados. No Brasil, trabalho realizadoem pacientes portadores de dermatoses diversas, mostrou uma taxa de positividade de $37,50 \%$, duas vezes menor que a encontrada neste trabalho ${ }^{13}$. A diferença de metodologia talvez possa explicar também esta diferença nos resultados, já que além de usarmos o extrator de cravos fizemos a colheita do material em toda extensão da face dos indivíduos examinados. A extração manual e raspagem da face para coleta, não é o método mais apropriado para obtenção do material, pois se sabe que a melhor extração se obtem com o extrator apropriado. Na Venezuela, entre indivíduos escolhidos ao acaso, a taxa de positividade obtida foide $39,13 \%$ para o parasitismo na região ocular ${ }^{3}$. Já na Dinamarca, estudo utilizando o mesmo procedimento, encontrou $48 \%$ de indivíduos positivos ${ }^{7}$.

A alta prevalência de ácaros demonstrada no presente trabalho para as faixas etárias iniciais, e o decréscimo discreto do parasitismo após os 40 anos, mostra-se em desacordo com a maioria dos dados da literatura ${ }^{7} 101114$. Entretanto, alguns trabalhos referem resultados semelhantes aos obtidos por nós. No Brasil, foi verificada uma taxa elevada de parasitismo entre indivíduos de 11 a 20 anos (42\%) a qual foi aumentando discretamente até os 50 anos, seguida de uma queda acentuada após essa idade ${ }^{13}$. Na Nigéria, também a prevalência nas faixas etárias mais jovens foi alta, seguida de uma queda acentuada $(3,3 \%)$ acima dos $30 \operatorname{anos}^{2}$. A alta prevalência obtida em nosso trabalho para as faixas iniciais de idade talvez possa ser explicada pelo número desigual de indivíduos dentro de cada faixa etária, já que os dois grupos etários iniciais compreendiam mais de $60 \%$ do total amostral; ou porque a amostra era de pessoas que procuraram uma dermo-estética, devido a problemas de pele. Este último fato pode estar favorecendo esta alta prevalência e sugere por sua vez que a existência de ácaros na pele possivelmente tem alguma relação com a procura desses cuidados.

Para explicar o baixo parasitismo de cinco tribos da África, foi aventada a hipótese de que altas temperaturas e insolação inibiriam a propagação de ácaros ${ }^{2}$. Entretanto, os resultados obtidos neste trabalho contradizem esta opinião e indicam que em países quentes há possibilidade de existir diferenças na prevalência para os grupos etários mais jovens, que apresentam um parasitismo mais elevado em relação ao verificado nos países temperados.

Quanto ao sexo, o parasitismo foi maior no sexo feminino em relação ao masculino, $74,4 \% \mathrm{e}$ $50 \%$ respectivamente. Esta diferença pode estar relacionada ao pequeno número de homens na amostra. Prevalências semelhantes para ambos os sexos têm sido observadas ${ }^{716}$ e também tendendo para um dos $\operatorname{sexos}^{13}$. Estes últimos autores acreditam não haver influência do sexo na distribuição do parasita, embora sugiram que a influência hormonal sobre as glândulas sebáceas possam causar alguma mudança na prevalência do $D$. brevis.

Não foi possível fazer nenhuma correlação entre o fator raça e prevalência dos demodicideos, pois o parasitismo se apresentou apenas nos indíviduos de pele clara, sendo que os 3 descendentes de japoneses pesquisados foram negativos. $O$ parasitismo nas mais diferentes raças tem sido relatado $^{9}$, sendo que nos Estados Unidos a prevalência foi igual para negros e brancos 14 .

Não conseguimos estabelecer uma relação entre presença de ácaros e lesões tipo comedon e acne, 
Madeira NG, Sogayar MIL. Prevalência de Demodex folliculorum e Demodex brevis em uma amostra da população de Botucatu, São Paulo, Brasil. Revista da Sociedade Brasileira de Medicina Tropical 26:221-224, out-dez, 1993.

pois até os indivíduos negativos tinham acne e comedon. A mesma dificuldade se apresentou com os indivíduos que tinham somente acne, pois $36,37 \%$ dessa amostra se apresentou negativo para o ácaro.

As amostragens dispares obtidas quanto ao tipo de pele, não possibilitaram correlações com a presença de ácaros pois dos 72 individuos parasitados, 70 tinham pele oleosa e 2 tinham pele normal. É conhecido, no entanto, o fato de que a pele oleosa predispõe os indivíduos ao parasitismo por ácaros.

\section{SUMMARY}

A survey was conduced to determine the prevalence of $\mathrm{D}$. folliculorum and $\mathrm{D}$. brevis for the first time in Brazil. In this study, association between the presence of mites and host factors (age, sex and race) were analysed. Samples were obtained from 100 individuals submitted to a facial cleaning in an aesthetic clinic in Botucatu city. All the samples were mounted in Berleses medium and examined by dark-field phase microscopy. From 100 studied individuals, 72 were positive. among the positive cases, $51 \%$ showed D. folliculorum, $2 \%$ showed D. brevis and $19 \%$ both species. The parasite distribution in realtion to sex was not taken in account because the sex ratio tavoured females (90\%). According to age, prevalence was high in all age groups. The factors influencing this distribution could be due. 1. the examination of extensive skin areas, 2 . the group examined composed of individuals in treatment in an anaesthetic clinic could be more infested than a normal population, 3. the fact that in tropics, the prevalence is often high in all ages.

Key-words: Demodex folliculorum. Demodex brevis. Prevalence.

\section{AGRADECIMENTOS}

À esteticista Cristina Ap. Belvel Fernandes Lastoria, da Dermo-Estética-Botucatu, pela coleta do material.

\section{REFERÊNCIAS BIBLIOGRÁFICAS}

1. Ayres S. Demodectic eruptions (demodicidosis) in the human. Archives of Dermatology 83:816-27, 1961.

2. Bozdech V, Moronfoye LS. Incidence of Demodex folliculorum in normal urban population in Kaduna (Nigeria). Folia Parasitologica 20:179-82, 1973.

3. Cordero-Moreno R, Ganteaume BC. Blefaritis acaria. Gaceta Medica de Caracas 97:121-3, 1989.

4. Desch C, Nutting WB. Demodex folliculorum(Simon) and $D$. brevis Akbulatova of man: Redescription and reevaluation. Journal of Parasitology 58:169-177, 1972.

5. Dominey A, Rosen T, Tschen J. Papulonodular Demodicidosis Associated with Acquired immunodeficiency Syndrome. Journal of the American Academy of Dermatology 20:197-201, 1989.

6. Huismans H. Demodex folliculorum. Klinische Monatsblaetter Fuer Augenheilkunde 193:304 5, 1988.

7. Norn MS. Demodex folliculorum. Incidence and possible pathogenic role in human eyelid. Acta Ophthalmologica 108:77-85, 1970.

8. Nunzi E, Rebora A, Hamgrlinck F, Cormane RH. Immunopathological studies on rosacea. British Journal of Dermatology 103:543-551, 1980.

9. Nutting WB. Hair follucle mites (Acari: Demodicidae) of man. International Journal of Dermatology 15:79-97, 1976.

10. Orru A, Pintus ML, Pinetti P. Studio sulla infestazione de Demodex folliculorum della cute umana normale. Rassegna Medica Sarda 75:231-244, 1972.

11. Post C, Juhlin E. Demodex folliculorum and blepharitis. Archives of Dermatology Syphilology 88:298-302, 1963.

12. Ramelet AA, Perrolaz G. Rosacee: étude histopathologique de 75 cas. Annales de Dermatologie et de Venereologie 115:801-6, 1988.

13. Santos I, Araújo TC, Neto FC, Lima MB. Contribuição ao estudo da demodicidosis. Anais Brasileiros de Dermatologia 58:205-208, 1983.

14. Sengbuch HG, Hauswirth JW. Prevalence of hair follicle mite, Demodex folliculorum and D.brevis (Acari: Demodicidae), in selected human population in western New York, USA. Journal of Medical Entomology 23:384-388, 1986.

15. Shelley WB, Shelley ED, Burmeister V. Unilateral demodectic rosacea. Journal of the American Academy of Dermatology 20:915-917, 1989.

16. Sibenge S, Gawkrodger DJ. Rosacea: A study of clinical patterns, blood flow, and the role of Demodex folliculorum. Journal of the Americam Academy of Dermatology 26:590-593, 1992.

17. Wolf R, Ophir J, Avigad J, Lengy J, Krakowski A. The hair follice mites (Demodex ssp). Could they be vectors of pathogenic microorganisms? Acta Dermato-Venereologica 68:535-537, 1988. 